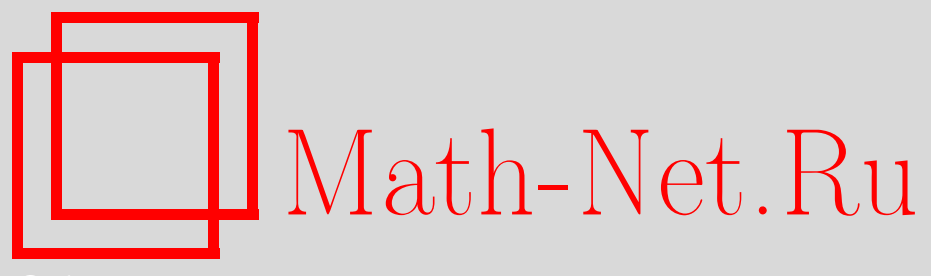

Б. Г. Конопельченко, Ф. Магри, Бездисперсионные интегрируемые уравнения как коизотропные деформации. Обобщения и редукции, ТМФ, 2007, том 151, номер 3, 439-457

DOI: https://doi.org/10.4213/tmf6058

Использование Общероссийского математического портала Math-Net.Ru подразумевает, что вы прочитали и согласны с пользовательским соглашением http: //www . mathnet.ru/rus/agreement

Параметры загрузки:

IP: 54.81 .137 .203

26 апреля 2023 г., 15:44:27

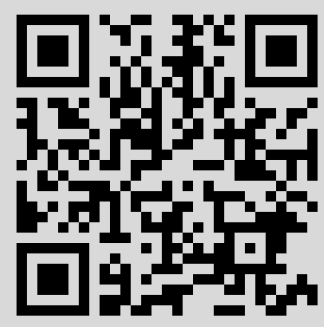




\title{
БЕЗДИСПЕРСИОННЫЕ ИНТЕГРИРУЕМЫЕ УРАВНЕНИЯ КАК КОИЗОТРОПНЫЕ ДЕФОРМАЦИИ. ОБОБЩЕНИЯ И РЕДУКЦИИ
}

\begin{abstract}
Обсуждается трактовка бездисперсионных интегрируемых иерархий как уравнений коизотропных деформаций для некоторых ассоциативных алгебр и других алгебраических структур. Показано, что в рамках такого подхода бездисперсионные уравнения Хироты для бездисперсионной иерархии КадомцеваПетвиашвили являются не чем иным, как условиями ассоциативности в некоторой параметризации. Рассмотрено несколько обобщений. Доказано, что бездисперсионные интегрируемые иерархии типа Б, подобно бездисперсионной иерархии Кадомцева-Петвиашвили типа Б и бездисперсионной иерархии ВеселоваНовикова, представляют собой коизотропные деформации тройных систем Йордана. Показано, что стационарные редукции бездисперсионных интегрируемых уравнений связаны с динамическими системами на плоскости, вполне интегрируемыми на фиксированном уровне энергии.
\end{abstract}

Ключевые слова: ассоциативные алгебры, коизотропные деформации, интегрируемые уравнения.

\section{1. ВВЕДЕНИЕ В ТЕОРИЮ КОИЗОТРОПНЫХ ДЕФОРМАЦИЙ}

На протяжении двух последних десятилетий бездисперсионные интегрируемые уравнения и иерархии привлекали к себе значительное внимание (см., например, работы [1]-[10]). Основным примером таких уравнений является бездисперсионное уравнение Кадомцева-Петвиашвили (уравнение бКП)

$$
\begin{aligned}
\frac{\partial u}{\partial x_{3}} & =\frac{3}{2} u \frac{\partial u}{\partial x_{1}}+\frac{\partial v}{\partial x_{2}} \\
\frac{\partial v}{\partial x_{1}} & =\frac{3}{4} \frac{\partial u}{\partial x_{2}} .
\end{aligned}
$$

Это уравнение эквивалентно условию совместности

$$
\frac{\partial^{2} S}{\partial x_{2} \partial x_{3}}=\frac{\partial^{2} S}{\partial x_{3} \partial x_{2}}
$$

*Dipartimento di Fisica, Universita' di Lecce and Sezione INFN, 73100 Lecce, Italy. E-mail: konopel@le.infn.it Italy

†Dipartimento di Matematica ed Applicazioni, Universita' di Milano Bicocca, 20126 Milano, 
для пары уравнений

$$
\begin{aligned}
\frac{\partial S}{\partial x_{2}} & =\left(\frac{\partial S}{\partial x_{1}}\right)^{2}+u \\
\frac{\partial S}{\partial x_{3}} & =\left(\frac{\partial S}{\partial x_{1}}\right)^{3}+\frac{3}{2} u \frac{\partial S}{\partial x_{1}}+v .
\end{aligned}
$$

Высшие уравнения бКП возникают как условия совместности для уравнения (1.2) и уравнений (см. работы [1]--[7])

$$
\frac{\partial S}{\partial x_{n}}=\left(\frac{\partial S}{\partial x_{1}}\right)^{n}+\sum_{m=0}^{n-2} u_{n m}(x)\left(\frac{\partial S}{\partial x_{1}}\right)^{m} .
$$

Другие бездисперсионные интегрируемые уравнения обычно ассоциируются с уравнениями типа Гамильтона-Якоби вида [6], [7]

$$
\frac{\partial S}{\partial x_{n}}=\Omega_{n}\left(\frac{\partial S}{\partial x_{1}}, x\right)
$$

где $\Omega_{n}$ - некоторые мероморфные функции первого аргумента.

В этой традиционной постановке переменные $x_{1}$ и $p=\partial S / \partial x_{1}$ играют выделенную роль. Они образуют пару канонически сопряженных переменных, и условия совместности для уравнений (1.5) могут быть записаны в виде

$$
\frac{\partial \Omega_{n}}{\partial x_{m}}(p, x)-\frac{\partial \Omega_{m}}{\partial x_{n}}(p, x)-\left\{\Omega_{n}(p, x), \Omega_{m}(p, x)\right\}=0,
$$

где скобка Пуассона $\{\cdot, \cdot\}$ определена следующим образом:

$$
\{f, g\}=\frac{\partial f}{\partial x_{1}} \frac{\partial g}{\partial p}-\frac{\partial f}{\partial p} \frac{\partial g}{\partial x_{1}} .
$$

Таким образом, в целом при обычном подходе мы имеем дело с нестационарными уравнениями Гамильтона-Якоби (1.5) и гамильтонианом $\Omega_{n}(p, x)$.

В настоящей работе будет рассмотрен новый подход к бездисперсионным интегрируемым уравнениям и иерархиям, при котором они представляют собой коизотропные деформации ассоциативных алгебр и других алгебраических структур [11].

Исходным пунктом этого подхода, представляющего собой сплав идей, позаимствованных из гамильтоновой механики и теории ассоциативных алгебр, является представление уравнений Гамильтона-Якоби (1.5) как стационарных уравнений. В рамках такого представления естественно рассматривать все переменные $x_{1}, x_{2}$, $x_{3}, \ldots$ на единой основе и ввести соответствующие канонические переменные $p_{1}, p_{2}$, $p_{3}, \ldots$, сопряженные переменным $x_{1}, x_{2}, x_{3}, \ldots$ относительно стандартной скобки Пуассона $\{\cdot, \cdot\}$, заданной выражением

$$
\{f, g\}=\sum_{i=1}^{n}\left(\frac{\partial f}{\partial x_{i}} \frac{\partial g}{\partial p_{i}}-\frac{\partial f}{\partial p_{i}} \frac{\partial g}{\partial x_{i}}\right) .
$$


Таким образом, мы вводим симплектическое многообразие $M^{2 n}$, снабженное скобкой Пуассона (1.8). Уравнения Гамильтона-Якоби (1.5) заменяются условиями обращения в нуль (нулевым множеством) гамильтонианов

$$
h_{n} \equiv-p_{n}+\Omega_{n}\left(p_{1}, x\right)=0 .
$$

Подчеркнем, что теперь $x_{i}, p_{i}$ образуют пары канонически сопряженных переменных, и $p_{i} \neq \partial S / \partial x_{i}$. Будем обозначать подмногообразие в $M^{2 n}$, заданное нулевым множеством (1.9), через $Г$.

Так, например, вместо уравнений (1.2), (1.3) имеем теперь нулевое множество гамильтонианов

$$
\begin{aligned}
& h_{2}=-p_{2}+p_{1}^{2}+u\left(x_{1}, x_{2}, x_{3}\right), \\
& h_{3}=-p_{3}+p_{1}^{3}+\frac{3}{2} u p_{1}+v .
\end{aligned}
$$

Подмногообразие $Г$, определенное уравнениями (1.10), “параметризуется” функциями $u$ и $v$ координат $x_{1}, x_{2}, x_{3}$.

Второй шаг в нашем подходе заключается в том, чтобы ввести особый класс подмногообразий Г. Естественно потребовать, чтобы гамильтоновы потоки, порожденные гамильтонианами $h_{2}$ и $h_{3}$, сохраняли $\Gamma$, или, другими словами, чтобы гамильтоновы векторные поля, ассоциированные с $h_{2}$ и $h_{3}$, являлись касательными к подмногообразию Г. Данное требование эквивалентно условию

$$
\left.\left\{h_{2}, h_{3}\right\}\right|_{\Gamma}=0 .
$$

Нетрудно проверить, что это условие выполнено тогда и только тогда, когда функции $u$ и $v$ удовлетворяют уравнению бКП (1.1). В этом случае $\left\{h_{2}, h_{3}\right\}=0$.

Таким образом, для любого решения $u, v$ уравнения бКП (1.1) гамильтонианы $h_{2}$ и $h_{3}$ находятся в инволюции, и деформация переменных $x_{1}, x_{2}, x_{3}$ гамильтоновыми потоками сохраняет подмногообразие Г.

Подобные деформации мы будем называть коизотропными по следующей причине. Подмногообразие Г, определяемое уравнениями (1.10) и удовлетворяющее указанным выше условиям, является 4-мерным подмногообразием в $M^{6}$, для которого $\left.\left\{h_{2}, h_{3}\right\}\right|_{\Gamma}=0$, и, более того, сужение стандартной симплектической 2-формы

$$
\omega \equiv \sum_{i=1}^{3} d p_{i} \wedge d x_{i}
$$

на подмногообразие Г не обращается в нуль тождественно. Поскольку ядро формы $\omega$ натягивается на гамильтоновы векторные поля, ассоциированные с гамильтонианами $h_{2}$ и $h_{3}$, то ранг сужения $\left.\omega\right|_{\Gamma}$ равен двум, и, следовательно,

$$
\omega_{\Gamma}=d \mathcal{L} \wedge d \mathcal{M}
$$

где $\mathcal{L}$ и $\mathcal{M}$ - канонические переменные на подмногообразии Г. Подмногообразия с такими свойствами называются коизотропными подмногообразиями (см., например, работы [12]). 
Итак, любое решение $u, v$ уравнения бКП посредством условий (1.10) определяет 4-мерное коизотропное подмногообразие в $M^{6}$, и в силу этого естественно называть соответствующие деформации коизотропными.

Аналогично условия $\left\{h_{n}, h_{m}\right\}=0$ для гамильтонианов $h_{n}$, заданных формулой (1.9), определяют коизотропные деформации. Нетрудно проверить, что условия коизотропности для гамильтонианов (1.9) в точности совпадают с условиями (1.6). В частности, для полиномиальных $\Omega_{n}\left(p_{1}\right)$ типа (1.10) коизотропные деформации даются бездисперсионной иерархией Кадомцева-Петвиашвили (иерархией бКП).

Переформулировка бездисперсионных интегрируемых уравнений и иерархий на языке коизотропных деформаций не только приводит к техническим упрощениям, но и открывает путь для существенных обобщений и новой интерпретации [11].

В основе значительного расширения предложенного подхода лежит то наблюдение, что можно перейти от нулевого множества функций $h_{n}$ к порожденному ими идеалу $J$. Действительно, любая полиномиальная функция $f_{n}$ от $h_{1}, h_{2}, \ldots$ принадлежит подмногообразию $\Gamma$, и условие коизотропности $\left.\left\{f_{n}, f_{m}\right\}\right|_{\Gamma}=0$ выполнено благодаря условию $\left.\left\{h_{n}, h_{m}\right\}\right|_{\Gamma}=0$. Более того, оба набора функций порождают одни и те же уравнения для потенциалов.

Таким образом, условие коизотропности принимает вид условия замкнутости идеала $J$ относительно скобки Пуассона (1.8), т.е.

$$
\{J, J\} \subset J .
$$

Переход к идеалу $J$ предоставляет свободу выбора в нем базиса разными способами. Рассмотрим для примера идеал, порожденный гамильтонианами уравнения бКП

$$
h_{0} \equiv-p_{0}+1
$$

и

$$
h_{n}=-p_{n}+p^{n}+\sum_{m=0}^{n-2} u_{n m}(x) p^{m}, \quad n=1,2,3, \ldots,
$$

где для удобства мы добавили гамильтонианы $h_{0}=-p_{0}+1$ и $h_{1}=-p_{1}+p$. Формально мы должны ввести переменную $x$, сопряженную переменной $p$. Это означает, что функции $u_{n m}$ будут зависеть только от $x_{1}+x$. Прежде всего, поскольку $-p_{2}+p_{1}^{2}+u=0$ на $\Gamma$, имеем $u=p_{2}-p_{1}^{2}$. Подставляя это выражение для $u$ в выражение для $h_{3}$, получаем новый гамильтониан

$$
\tilde{h}_{3}=-p_{3}-\frac{1}{2} p_{1}^{3}+\frac{3}{2} p_{1} p_{2}+v .
$$

Продолжая подобную процедуру далее, мы получаем бесконечное множество гамильтонианов $\tilde{h}_{n}$, которые имеют вид суммы полиномов по переменным $p_{1}, p_{2}, \ldots$ и функций $u_{n}\left(x_{1}, x_{2}, \ldots\right)$, а именно

$$
\tilde{h}_{n}=n P_{n}(\tilde{p})+u_{n}(x), \quad n=2,3, \ldots,
$$

где $\tilde{p}=\left(-p_{1},-p_{2} / 2,-p_{3} / 3, \ldots\right), u_{2}=u_{20}=u, u_{3}=u_{30}=v$ и $P_{n}(t)-$ полиномы Шура, определяемые формулой

$$
\exp \left(\sum_{n=1}^{\infty} z^{n} t_{n}\right)=\sum_{m=0}^{\infty} z^{m} P_{m}(t)
$$


Гамильтонианы $\tilde{h}_{n}$ вместе с гамильтонианами $h_{0}$ и $h_{1}$ образуют базис в том же идеале $J$, который порождается функциями $h_{n}(1.15)$.

Для гамильтонианов (1.17) условие коизотропности (1.14) означает, что

$$
\left\{\tilde{h}_{n}, \tilde{h}_{m}\right\}=\sum_{k=1}^{n-2} \frac{n}{k(n-k)} \frac{\partial u_{m}}{\partial x_{k}} \tilde{h}_{n-k}-\sum_{k=1}^{m-2} \frac{m}{k(m-k)} \frac{\partial u_{n}}{\partial x_{k}} \tilde{h}_{m-k}
$$

и верны следующие уравнения:

$$
\begin{gathered}
\frac{n-1}{n} \frac{\partial u_{n}}{\partial x_{m-1}}=\frac{m-1}{m} \frac{\partial u_{m}}{\partial x_{n-1}}, \\
\frac{\partial u_{m}}{\partial x_{n}}-\frac{\partial u_{n}}{\partial x_{m}}+\sum_{k=1}^{m-2} \frac{m}{k(m-k)} u_{m-k} \frac{\partial u_{n}}{\partial x_{k}}-\sum_{k=1}^{n-2} \frac{n}{k(n-k)} u_{n-k} \frac{\partial u_{m}}{\partial x_{k}}=0
\end{gathered}
$$

где $m, n=2,3, \ldots$. Можно проверить, что уравнения (1.19) и (1.20) эквивалентны обычной иерархии бКП.

Интересный базис возникает при попытке преобразовать (1.15) в базис, билинейный по переменным $p_{1}, p_{2}, p_{3}, \ldots$. Он может быть легко построен следующим образом. Из первого уравнения (1.10) имеем $p_{1}^{2}=p_{2}-u$. Подставляя это выражение для $p_{1}^{2}$ во второе уравнение (1.10), получаем уравнение

$$
-f_{12} \equiv p_{1} p_{2}-p_{3}+\frac{1}{2} u p_{1}+v=0
$$

Затем, получая из второго уравнения (1.10) выражение $p_{1}^{3}=p_{3}-3 u p_{1} / 2-v$ и подставляя его и выражение $p_{1}^{2}=p_{2}-u$ в уравнение $(1.15)$ с $n=4$, приходим к уравнению

$$
-f_{13}=p_{1} p_{3}-p_{4}+\left(u_{42}-\frac{3}{2} u\right) p_{2}-v p_{1}+u_{40}-u u_{42}=0 .
$$

Повторяя подобную процедуру, мы получаем семейство гамильтонианов вида [11]

$$
-f_{j k} \equiv p_{j} p_{k}-\sum_{l=0}^{j+k} C_{j k}^{l}(x) p_{l}=0, \quad j, k,=1,2,3, \ldots
$$

где $C_{j k}^{l}$ - некоторые коэффициенты и $p_{0} \equiv 1$. Знак минус в левой части последней формулы выбран для того, чтобы функции $f_{j k}$ были теми же, что и в работе [11]. Соотношение

$$
f_{j k}=h_{j} h_{k}-\sum_{l \geqslant 0} C_{j k}^{l}(x) h_{l}+p_{j} h_{k}+p_{k} h_{j}
$$

показывает, что нулевое множество гамильтонианов $h_{k}$ совпадает с нулевым множеством гамильтонианов $f_{j k}$. Прямые (хотя и громоздкие) вычисления показывают, что условия коизотропности для гамильтонианов $f_{j k}$ снова дают начало иерархии бКП. 
Таким образом, выбор базиса в идеале $J$ не влияет на условия коизотропности. Интересно, однако, что указанная свобода позволяет обнаружить глубокие алгебраические корни бездисперсионных интегрируемых уравнений. Действительно, уравнения (1.23) весьма похожи на реализацию таблицы умножения для ассоциативной алгебры в базисе, образованном переменными $p_{0}, p_{1}, p_{2}, \ldots$. Это наблюдение приводит к возможности трактовать иерархию бКП как коизотропные деформации коммутативной ассоциативной алгебры [11].

Именно, пусть мы имеем коммутативную ассоциативную алгебру. Выберем в алгебре базис $p_{0}(\equiv 1), p_{1}, p_{2}, \ldots$ и, таким образом, получим соответствующую таблицу умножения

$$
p_{j} p_{k}=\sum_{l=0} C_{j k}^{l} p_{l} .
$$

Коммутативность означает, что $C_{j k}^{l}=C_{k j}^{l}$, а из ассоциативности следует

$$
\sum_{l=0} C_{j k}^{l} C_{l m}^{p}=\sum_{l=0} C_{m k}^{l} C_{l j}^{p}
$$

Чтобы рассмотреть деформации, предположим, что структурные константы $C_{j k}^{k}$ зависят от набора переменных $x_{1}, x_{2}, x_{3}, \ldots$. Для определения деформации свяжем с таблицей умножения (1.24) набор квадратичных гамильтонианов

$$
f_{j k}=-p_{j} p_{k}+\sum_{l=0} C_{j k}^{l}(x) p_{l},
$$

где $x_{j}, p_{j}$ образуют пары канонически сопряженных переменных в симплектическом многообразии $M$, снабженном скобкой Пуассона (1.8).

Рассмотрим затем полиномиальный идеал $J=\left\langle f_{j k}\right\rangle$, порожденный этими гамильтонианами, и подмногообразие $Г$, определяемое формулой

$$
\Gamma=\left\{\left(x_{j}, p_{j} \in M ; f_{j k}=0\right)\right\} .
$$

Наконец, если идеал $J$ замкнут относительно скобки Пуассона, т.е.

$$
\{J, J\} \subset J,
$$

так что Г является коизотропным подмногообразием в $M$, то говорят, что функции $C_{j k}^{l}(x)$ определяют коизотропную деформацию ассоциативной алгебры (1.24) [11].

Можно показать, что условие (1.28) выполнено, если структурные константы $C_{j k}^{l}$ удовлетворяют системе уравнений

$$
\sum_{s=1}\left(C_{s j}^{m} \frac{\partial C_{l r}^{s}}{\partial x_{k}}+C_{s k}^{m} \frac{\partial C_{l r}^{s}}{\partial x_{j}}-C_{s r}^{m} \frac{\partial C_{j k}^{s}}{\partial x_{l}}-C_{s l}^{m} \frac{\partial C_{j k}^{s}}{\partial x_{r}}+\frac{\partial C_{j k}^{m}}{\partial x_{s}} C_{l r}^{s}-\frac{\partial C_{l r}^{m}}{\partial x_{s}} C_{j k}^{s}\right)=0 .
$$

Система уравнений (1.25), (1.29) полностью определяет коизотропные деформации ассоциативной алгебры (1.24) и составляет основу нашего подхода [11]. По этой причине мы будем называть эту систему основной системой теории коизотропных деформаций.

Подчеркнем, что основная система - это система алгебраических и дифференциальных уравнений исключительно для структурных констант $C_{j k}^{l}$. Мы увидим, что данная особенность является существенным преимуществом рассматриваемого здесь подхода. 


\section{2. КОИЗОТРОПНЫЕ ДЕФОРМАЦИИ ТИПА БКП: ТАУ-ФУНКЦИЯ И УРАВНЕНИЯ ХИРОТЫ}

Центральной проблемой, связанной с основной системой (1.25), (1.29), является, разумеется, существование и смысл ее нетривиальных решений. Решение дается иерархией бКП, для которой структурные константы $C_{j k}^{l}$ могут быть восстановлены через потенциалы $u_{n m}(x)$ формулы (1.15) посредством формулы (1.23).

С другой стороны, анализ уравнений (1.23) показывает [11], что структурные константы $C_{j k}^{l}$ иерархии бКП имеют следующий общий вид:

$$
C_{k j}^{l}=\delta_{k+j}^{l}+H_{j-l}^{k}+H_{k-l}^{j},
$$

где $k, j, l=0,1,2,3, \ldots, \delta_{k}^{l}$ - символ Кронекера и $H_{j}^{k}$ - функции переменных $x_{1}, x_{2}, \ldots$ такие, что $H_{0}^{k}=0$ и $H_{j}^{k}=0$ для $j \leqslant-1$. Мы покажем, что использование структурных констант общего (не параметризированного) вида (2.1) в основной системе приводит к существованию тау-функции и новой алгебраической интерпретации бездисперсионных уравнений Хироты [11].

Первый шаг состоит в том, чтобы обеспечить выполнение условий ассоциативности в терминах коэффициентов $H_{j}^{k}$. Прямая подстановка выражений $(2.1)$ в уравнения (1.25) и использование тождества

$$
\sum_{l=n-1}^{k-1} H_{k-l}^{i} H_{l-n}^{m}=\sum_{l=n-1}^{k-1} H_{k-l}^{m} H_{l-n}^{i}
$$

показывают, что структурные константы $C_{j k}^{l}$ удовлетворяют условиям ассоциативности тогда и только тогда, когда скобка

$$
[H, H]_{i k m}:=H_{m}^{i+k}-H_{m+k}^{i}-H_{i+m}^{k}+\sum_{l=1}^{i-1} H_{i-l}^{k} H_{m}^{l}+\sum_{l=1}^{k-1} H_{k-l}^{i} H_{m}^{l}-\sum_{l=1}^{m-1} H_{m-l}^{k} H_{l}^{i}
$$

тождественно обращается в нуль при любом выборе индексов $(i, k, m) \in N$. Интересное следствие этого результата может быть выведено путем свертывания индексов. Действительно, можно проверить, что следствием приведенных выше уравнений являются полезные соотношения симметрии

$$
p H_{p}^{i}=i H_{i}^{p} .
$$

Далее, необходимо обеспечить выполнение условий коизотропности. Получающиеся уравнения в терминах коэффициентов $H_{k}^{i}$ достаточно сложны, однако более тщательное рассмотрение показывает, что они радикально упрощаются, если учесть только что полученные условия ассоциативности. Действительно, можно доказать, что условия коизотропности могут быть сведены к уравнениям

$$
\frac{\partial[H, H]_{l, n, i-j}}{\partial x_{k}}+\frac{\partial[H, H]_{l, n, k-j}}{\partial x_{i}}-\frac{\partial[H, H]_{i, k, l-j}}{\partial x_{n}}-\frac{\partial[H, H]_{i, k, n-j}}{\partial x_{l}}=0,
$$

которые автоматически выполняются при наличии условий ассоциативности, и к линейным уравнениям

$$
\frac{\partial H_{p}^{i}}{\partial x_{l}}=\frac{\partial H_{p}^{l}}{\partial x_{i}}
$$


Итак, в случае структурных констант вида (2.1) условия ассоциативности и коизотропности вместе эквивалентны набору квадратичных алгебраических уравнений

$$
[H, H]_{i k l}=0
$$

и набору линейных дифференциальных уравнений

$$
\frac{\partial H_{p}^{i}}{\partial x_{l}}=\frac{\partial H_{p}^{l}}{\partial x_{i}}
$$

имеющих вид системы законов сохранения. Уравнения (2.4) и (2.5) дают конкретный вид основной системы для иерархии бКП. Эта система содержит в закодированном виде всю информацию об иерархии. Из нее, в частности, вытекает, что для любого решения основной системы имеет место формула

$$
\left\{f_{i k}, f_{l n}\right\}=\sum_{s, t \geqslant 1} K_{i k l n}^{s t} f_{s t},
$$

где

$$
K_{i k l n}^{s t}=\left(\delta_{i t} \frac{\partial}{\partial x_{k}}+\delta_{k t} \frac{\partial}{\partial x_{i}}\right)\left(H_{l-s}^{n}+H_{n-s}^{l}\right)-\left(\delta_{n t} \frac{\partial}{\partial x_{l}}+\delta_{l t} \frac{\partial}{\partial x_{n}}\right)\left(H_{i-s}^{k}+H_{k-s}^{i}\right) .
$$

Из этой формулы видно, что гамильтонианы $f_{j k}$ иерархии бКП образуют алгебру Пуассона. Упомянутая выше основная система может рассматриваться также как бездисперсионный предел основной системы для полной дисперсионной иерархии Кадомцева-Петвиашвили [13].

Существуют две стратегии по раскодированию информации, содержащейся в основной системе. Согласно первой мы сначала пытаемся разрешить условия ассоциативности $(2.4)$, замечая, что они позволяют вычислить коэффициенты $\left(H_{k}^{2}, H_{k}^{3}, \ldots\right)$ в виде полиномиальных функций от $H_{k}^{1}$. Например, условия симметрии

$$
H_{1}^{2}=2 H_{2}^{1}, \quad H_{1}^{3}=3 H_{3}^{1}
$$

дают $\left(H_{1}^{2}, H_{1}^{3}\right)$, а затем условие $(2.4)$ с $i=k=1, l=2$, т.е.

$$
H_{1}^{3}-H_{3}^{1}-H_{2}^{2}+H_{1}^{1} H_{1}^{1}=0
$$

дает $H_{2}^{2}$, и т.д. Переименовывая свободные коэффициенты в соответствии с таблицей умножения предыдущего раздела, т.е. полагая

$$
H_{1}^{1}=-\frac{1}{2} u, \quad H_{2}^{1}=-\frac{1}{3} v, \quad H_{3}^{1}=-\frac{1}{4} w+\frac{1}{8} u^{2},
$$

получаем

$$
H_{1}^{2}=-\frac{2}{3} v, \quad H_{2}^{2}=-\frac{1}{2} w+\frac{1}{2} u^{2}, \quad H_{1}^{3}=-\frac{3}{4} w+\frac{3}{8} u^{2} .
$$

Подставляя теперь эти выражения в простейшие линейные условия коизотропности

$$
\frac{\partial H_{1}^{1}}{\partial x_{2}}-\frac{\partial H_{1}^{2}}{\partial x_{1}}=0, \quad \frac{\partial H_{2}^{1}}{\partial x_{2}}-\frac{\partial H_{2}^{2}}{\partial x_{1}}=0, \quad \frac{\partial H_{1}^{1}}{\partial x_{3}}-\frac{\partial H_{1}^{3}}{\partial x_{1}}=0,
$$


приходим к уравнениям

$$
\begin{aligned}
\frac{\partial v}{\partial x_{1}} & =\frac{3}{4} \frac{\partial u}{\partial x_{2}}, \\
\frac{\partial v}{\partial x_{2}} & =\frac{3}{2} \frac{\partial w}{\partial x_{1}}-3 u \frac{\partial u}{\partial x_{1}}, \\
\frac{\partial u}{\partial x_{3}} & =\frac{3}{2} \frac{\partial w}{\partial x_{1}}-\frac{3}{2} u \frac{\partial u}{\partial x_{1}} .
\end{aligned}
$$

Исключение членов, содержащих $\partial w / \partial x_{1}$, приводит в конечном счете к уравнению бКП и к высшим уравнениям, если проявить достаточную настойчивость при вычислениях. Используя данную стратегию, мы возвращаемся к иерархии в ее обычной формулировке.

Простое изменение порядка рассмотрения уравнений приводит вместо этого к представлению Хироты. Достаточно заметить, что из уравнений (2.5) вытекает существование последовательности потенциалов $S_{m}$ таких, что

$$
H_{m}^{i}=\frac{\partial S_{m}}{\partial x_{i}} .
$$

Тогда условия симметрии (2.3) налагают на потенциалы $S_{m}$ следующие ограничения:

$$
i \frac{\partial S_{i}}{\partial x_{l}}=l \frac{\partial S_{l}}{\partial x_{i}}
$$

которые, в свою очередь, приводят к существованию суперпотенциала $F\left(x_{1}, x_{2}, \ldots\right)$ такого, что

$$
S_{i}=-\frac{1}{i} \frac{\partial F}{\partial x_{i}}, \quad H_{m}^{i}=-\frac{1}{m} \frac{\partial^{2} F}{\partial x_{i} \partial x_{m}} .
$$

Данный результат дает новую по сравнению с описанной выше параметризацию структурных констант. Подстановка этой параметризации в полный набор условий ассоциативности приводит в конечном счете к следующей системе уравнений:

$$
\begin{aligned}
-\frac{1}{m} F_{i+k, m} & +\frac{1}{m+k} F_{i, k+m}+\frac{1}{i+m} F_{k, i+m}+ \\
+ & \sum_{l=1}^{i-1} \frac{1}{m(i-l)} F_{k, i-l} F_{l, m}+\sum_{l=1}^{k-1} \frac{1}{m(k-l)} F_{i, k-l} F_{l, m}- \\
& -\sum_{l=1}^{m-1} \frac{1}{i(m-l)} F_{k, m-l} F_{i, l}=0,
\end{aligned}
$$

где $F_{i, k}$ обозначает производную второго порядка потенциала $F$ по переменным $x_{i}$ и $x_{k}$. Эти уравнения эквивалентны знаменитым билинейным уравнениям Хироты для тау-функции иерархии бКП (см., например, работы [4], [5], [8]). Для $i=k=1$, $m=2$, или $i=m=1, k=2$, или $m=k=1, i=2$, например, мы получаем первое уравнение Хироты

$$
-\frac{1}{2} F_{2,2}+\frac{2}{3} F_{1,3}-\left(F_{1,1}\right)^{2}=0 .
$$


Для $i=1, k=2, m=2$, или $i=2, k=1, m=2$, или $i=1, k=1, m=3$ взамен второго уравнения Хироты имеем

$$
\frac{1}{2} F_{1,4}-\frac{1}{3} F_{2,3}-F_{1,1} F_{1,2}=0 .
$$

Высшие уравнения (2.8) по отдельности не совпадают с билинейными уравнениями Хироты, однако вместе они эквивалентны обычным уравнениям Хироты того же веса.

Таким образом, бездисперсионные уравнения Хироты для иерархии бКП представляют собой не что иное, как условия ассоциативности (2.4) при параметризации (2.7). Подчеркнем, что данный результат является следствием использования структурных констант (2.1) вместо потенциалов $u_{n m}$ в основной системе.

Отметим, что функции $K_{i k l n}^{s t}$ в формуле (2.6) представляют собой линейную комбинацию производных третьего порядка от тау-функции $F$. Например,

$$
\left\{f_{11}, f_{1 n}\right\}=-2 \sum_{s=1}^{s=n-1}\left(\frac{1}{(n-s)} \frac{\partial^{3} F}{\partial x_{1}^{2} \partial x_{n-s}}\right) f_{1 s}, \quad n=2,3, \ldots
$$

Вероятно, появление третьих производных тау-функции как в уравнениях ВиттенаДийкграафа-Верлинде-Верлинде [8], [14], так и в уравнениях (2.9) в виде структурных констант не является простым совпадением.

Отметим также, что условия коизотропности (2.9) нарушаются в тех точках, где третья производная потенциала $F$ обращается в бесконечность. Эти точки отвечают сингулярному сектору иерархии бКП.

\section{3. РАСПРОСТРАНЕНИЕ МЕТОДА НА ДРУГИЕ ИЕРАРХИИ}

Несколько обобщений представленных в разделе 2 результатов связаны с достаточно очевидными расширениями определяемой формулой (1.15) полиномиальной алгебры.

Первое расширение получится, если ослабить условия для полиномов в правой части $(1.15)$, позволив им содержать члены вида $u_{n, n-1}(x) p^{n-1}$, т.е. рассматривать семейство гамильтонианов

$$
\begin{aligned}
& h_{0}=-p_{0}+1 \\
& h_{1}=-p_{1}+p \\
& \ldots \ldots \ldots \ldots \ldots \ldots \ldots \ldots \ldots \ldots \\
& h_{n}=-p_{n}+\sum_{m=0}^{n} u_{n m}(x) p^{m}, \quad n=2,3,4, \ldots,
\end{aligned}
$$

где $u_{n n}=1$. Условие коизотропности снова дается уравнением $\left\{h_{n}, h_{m}\right\}=0$, в котором скобка Пуассона определена следующим образом:

$$
\{f, g\}=\frac{\partial f}{\partial x} \frac{\partial g}{\partial p}-\frac{\partial f}{\partial p} \frac{\partial g}{\partial x}+\sum_{i=1}\left(\frac{\partial f}{\partial x_{i}} \frac{\partial g}{\partial p_{i}}-\frac{\partial f}{\partial p_{i}} \frac{\partial g}{\partial x_{i}}\right) .
$$


Переменная $x_{0}$, сопряженная переменной $p_{0}$, является циклической, и мы опускаем соответствующий член в формуле (3.2). В силу вида гамильтониана $h_{1}$ коэффициенты $u_{n m}$ зависят от суммы $x+x_{1}$.

Условия коизотропности для гамильтонианов (3.1) дают начало обобщенной иерархии бКП (см. работу [11]). В частном случае калибровки $u_{20}=0, u_{30}=0$ мы получаем бездисперсионную модифицированную иерархию Кадомцева-Петвиашвили (иерархию бмКП) [11].

Дальнейшее очевидное расширение состоит в том, чтобы позволить коэффициентам $u_{n n}$ зависеть от $x$. Соответствующие деформации содержат бездисперсионную иерархию Гарри Дима в качестве подкласса [15]. Действительно, выбирая

$$
\begin{aligned}
& h_{2}=-p_{2}+\rho^{2} p_{1}^{2}, \\
& h_{3}=-p_{3}+\rho^{3} p_{1}^{3}+\mu \rho^{2} p_{1}^{2},
\end{aligned}
$$

получаем из условия коизотропности систему

$$
\begin{aligned}
& \frac{\partial \rho^{2}}{\partial x_{3}}=\frac{\partial\left(\mu \rho^{2}\right)}{\partial x_{2}}, \\
& \frac{\partial \mu}{\partial x_{1}}=\frac{3}{2} \frac{\partial \rho}{\partial x_{2}},
\end{aligned}
$$

которая представляет собой $(2+1)$-мерное бездисперсионное уравнение Гарри Димa [15].

В рассмотренных выше расширениях мы оставались в классе полиномиальных алгебр с единственным генератором $p$. Обобщение всего подхода до полиномиальных алгебр с $n$ генераторами посредством процедуры склеивания было предложено в работе [11]. В простейшем случае двух генераторов $p$ и $q$ эта процедура заключается в добавлении к двум полиномиальным алгебрам (3.1) склеивающего соотношения

$$
p q=a p+b q+c .
$$

Соотношение (3.5) дает возможность построить полную таблицу умножений между элементами $p_{i}$ и $q_{k}$. В работе [11] было показано, что соответствующие коизотропные деформации даются двухточечной иерархией бКП, которая эквивалентна универсальной иерархии Уизема на римановой сфере с двумя выколотыми точками [6]. В частности, эта иерархия содержит простейшее $(2+1)$-мерное уравнение Бенни, введенное в работе [7].

Здесь мы хотели бы указать способ включения бездисперсионной двумерной цепочки Тоды (бДЦТ) в нашу схему. С этой целью рассмотрим две копии полиномиальных алгебр общего вида и, следовательно, два семейства гамильтонианов

$$
\begin{aligned}
& h_{0}=-p_{0}+1, \\
& h_{1}=-p_{1}+a p+b, \\
& \cdots \ldots \ldots \ldots \ldots \ldots \ldots \cdots \cdots \cdots \\
& h_{n}=-p_{n}+\sum_{m=0}^{n} u_{n m}(x, y) p^{m}
\end{aligned}
$$


и

$$
\begin{aligned}
& \tilde{h}_{0}=h_{0}=-p_{0}+1, \\
& \tilde{h}_{1}=-q_{1}+\tilde{a} q+\tilde{b}, \\
& \ldots \ldots \ldots \ldots \ldots \ldots \ldots \ldots \ldots \ldots \ldots \\
& \tilde{h}_{n}=-q_{n}+\sum_{m=0}^{n} \tilde{u}_{n m}(x, y) q^{m}
\end{aligned}
$$

со склеивающим соотношением

$$
f=p q-1=0 .
$$

Здесь $a, b, u_{n m}, \tilde{a}, \tilde{b}, \tilde{u}_{n m}$ - функции переменных $x, x_{1}, x_{2}, \ldots ; y, y_{1}, y_{2}, \ldots$, канонически сопряженных переменным $p, p_{1}, p_{2}, \ldots ; q, q_{1}, q_{2}, \ldots$.

Проанализируем теперь условие коизотропности для этих гамильтонианов. Нетрудно проверить, что использование канонической скобки Пуассона вида (1.7), например, для гамильтонианов $h_{1}, \tilde{h}_{1}, f$ приводит к слишком сильным ограничениям на функции $a, b, \tilde{a}, \tilde{b}$ и, как следствие, к тривиальным деформациям.

Однако если вместо этого использовать модифицированную скобку Пуассона, определенную следующим образом:

$$
\begin{aligned}
\{f, g\}=p & \left(\frac{\partial f}{\partial x} \frac{\partial g}{\partial p}-\frac{\partial f}{\partial p} \frac{\partial g}{\partial x}\right)-q\left(\frac{\partial f}{\partial y} \frac{\partial g}{\partial q}-\frac{\partial f}{\partial q} \frac{\partial g}{\partial y}\right)+ \\
& +\sum_{i=1}\left(\frac{\partial f}{\partial x_{i}} \frac{\partial g}{\partial p_{i}}-\frac{\partial f}{\partial p_{i}} \frac{\partial g}{\partial x_{i}}+\frac{\partial f}{\partial y_{i}} \frac{\partial g}{\partial q_{i}}-\frac{\partial f}{\partial q_{i}} \frac{\partial g}{\partial y_{i}}\right)
\end{aligned}
$$

то ситуация радикально меняется. Действительно, из условий $\left.\left\{h_{1}, f\right\}\right|_{\Gamma}=0$ и $\left.\left\{\tilde{h}_{1}, f\right\}\right|_{\Gamma}=0$ получаем

$$
\begin{array}{ll}
a_{x}-a_{y}=0, & b_{x}-b_{y}=0, \\
\tilde{a}_{x}-\tilde{a}_{y}=0, & \tilde{b}_{x}-\tilde{b}_{y}=0,
\end{array}
$$

в то время как условие $\left.\left\{h_{1}, \tilde{h}_{1}\right\}\right|_{\Gamma}=0$ дает

$$
\begin{aligned}
a_{y_{1}}+a \tilde{b}_{x} & =0, \\
\tilde{a}_{x_{1}}-\tilde{a} b_{y} & =0, \\
\tilde{a} a_{y}+a \tilde{a}_{x}+b_{y_{1}}-\tilde{b}_{x_{1}} & =0 .
\end{aligned}
$$

В частном случае калибровки $b=-a, \tilde{b}=-\tilde{a}$ из системы $(3.10),(3.11)$ следует, что

$$
\begin{aligned}
& a_{y_{1}}-a \tilde{a}_{x}=0, \\
& \tilde{a}_{x_{1}}+\tilde{a} a_{x}=0 .
\end{aligned}
$$

Из последней системы получаем

$$
\theta_{x_{1} y_{1}}+\left(e^{\theta}\right)_{x x}=0
$$


где $\theta=\ln (a \tilde{a})$. Это уравнение Бойера-Финли, или уравнение бДЦТ (см., например, работы [3], [5], [6]). Рассматривая условия коизотропности для высших гамильтонианов $h_{n}, \tilde{h}_{n}$, мы получаем высшие уравнения бДЦТ и всю иерархию бДЦТ в целом.

То обстоятельство, что для получения бДЦТ как коизотропных деформаций полиномиальных алгебр необходимо рассматривать модифицированную скобку Пуассона (3.9), заслуживает большого внимания. Оно указывает на то, что симплектическая структура деформаций должна быть согласована с алгеброй, которая подвергается деформации. Разумеется, можно легко превратить модифицированную скобку (3.9) в каноническую, если ввести переменную $w$ такую, что $p=e^{w}, q=e^{-w}$. Однако в терминах этой переменной гамильтонианы $h_{n}, \tilde{h}_{n}$ превращаются в полиномы экспонент $e^{w}$ и $e^{-w}$. Это явление, а также первая половина скобки (3.9) (при $q=1 / p)$ хорошо известны для иерархии бДЦТ (см., например, работы [3], [5], [6]).

Похожая ситуация - согласованность скобки Пуассона и вида гамильтонианов имеет место для бездисперсионных уравнений, рассмотренных в работе [16].

\section{4. КОИЗОТРОПНЫЕ ДЕФОРМАЦИИ ТРОЙНЫХ СИСТЕМ ЙОРДАНА: ИЕРАРХИИ ТИПА Б}

Совершенно другие интересные структуры появляются для “алгебр", совместимых с дискретной группой, т.е. в случае, когда дискретная группа нетривиально действует на элементы базиса, в то же время оставляя неизменными структурные константы. Здесь будет рассмотрен лишь простой пример группы одновременных отражений всех элементов базиса $p_{i} \rightarrow-p_{i}$.

Начнем с однокомпонентного случая. Естественный базис теперь задается нечетными степенями $p^{2 i+1}$, и аналоги полиномов (1.15) имеют вид

$$
\begin{aligned}
& p_{1}=p \\
& p_{3}=p^{3}+u p \\
& p_{5}=p^{5}+v_{3} p^{3}+v_{1} p \\
& p_{7}=p^{7}+w_{5} p^{5}+w_{3} p^{3}+w_{1} p
\end{aligned}
$$

и т.д. Такая алгебра, очевидно, не является замкнутой относительно обычной операции умножения $p_{i} p_{k}$. Однако соотношения

$$
\begin{aligned}
& p_{1}^{3}= p_{3}-u p_{1}, \\
& p_{1}^{2} p_{3}=p_{5}+\left(u-v_{3}\right) p_{3}-\left[u\left(u-v_{3}\right)+v_{1}\right] p_{1}, \\
& p_{1} p_{3}^{2}=p_{7}+\left(2 u-w_{5}\right) p_{5}+\left[u^{2}-w_{3}-v_{3}\left(2 u-w_{5}\right)\right] p_{3}+ \\
&+ {\left[\left(2 u-w_{5}\right)\left(v_{3} u-v_{1}\right)-u\left(u^{2}-w_{3}\right)-w_{1}\right] p_{1}, } \\
& p_{1}^{2} p_{5}=p_{7}+\left(v_{3}-w_{5}\right) p_{5}+\left[v_{1}-w_{3}-v_{3}\left(v_{3}-w_{5}\right)\right] p_{3}+ \\
&+\left[\left(v_{3}-w_{5}\right)\left(v_{3} u-v_{1}\right)-u\left(v_{1}-w_{3}\right)-w_{1}\right] p_{1}
\end{aligned}
$$

и аналогичные наводят на мысль о рассмотрении кубической операции $p_{i} p_{k} p_{l}$. Таким образом, нечетные полиномы (4.1) дают нам пример "алгебры" с базисом $p_{1}, p_{3}$, $p_{5}, \ldots$, замкнутой относительно коммутативной трилинейной операции

$$
p_{i} p_{k} p_{l}=\sum_{m=1}^{i+k+l} C_{i k l}^{m} p_{m},
$$


где все индексы принимают только нечетные целые значения и

$$
\begin{array}{ll}
C_{111}^{3}=1, & C_{111}^{1}=-u, \quad C_{113}^{5}=1, \\
C_{113}^{3}=u-v_{3}, & C_{113}^{1}=-\left[u\left(u-v_{3}\right)+v_{1}\right]
\end{array}
$$

и т.д. Алгебраические структуры, определенные трилинейным законом (4.3), известны как тройные системы Йордана (см., например, книгу [17]). Ассоциативность для тройной системы Йордана определяется как

$$
\left(p_{i} p_{k} p_{l}\right) p_{n} p_{m}=p_{i} p_{k}\left(p_{l} p_{n} p_{m}\right)=p_{i}\left(p_{k} p_{l} p_{n}\right) p_{m}
$$

что ведет к следующим условиям "ассоциативности":

$$
\begin{aligned}
& \sum_{t} C_{i k l}^{t} C_{t n m}^{s}=\sum_{t} C_{l n m}^{t} C_{i k t}^{s}, \\
& \sum_{t} C_{l n m}^{t} C_{i k t}^{s}=\sum_{t} C_{k l n}^{t} C_{i m t}^{s} .
\end{aligned}
$$

Рассмотренный выше подход может быть применен для определения коизотропных деформаций тройных систем Йордана. Итак, превратим таблицу умножения (4.3) в нулевое множество Г гамильтонианов

$$
f_{i k l}:=-p_{i} p_{k} p_{l}+\sum_{m=1} C_{i k l}^{m}(x) p_{m}, \quad i, k, l=1,3,5, \ldots,
$$

а затем потребуем коизотропности Г относительно канонической скобки Пуассона. Мы получим уравнения коизотропных деформаций для структурных констант $C_{i k l}^{m}$, аналогичные уравнениям (1.29).

Рассмотрим здесь простейшие из этих уравнений, возникающие как условия коизотропности для низших гамильтонианов:

$$
f_{111}=-p_{1}^{3}+p_{3}-u p_{1}, \quad f_{113}=-p_{1}^{2} p_{3}+p_{5}-v p_{3}-w p_{1} .
$$

Имеем

$$
\begin{aligned}
\left\{f_{111}, f_{113}\right\}= & \left(-u_{x_{2}}+3 w_{x_{1}}\right) f_{111}+\left(-2 u_{x_{1}}+3 v_{x_{1}}\right) f_{113}+\left(-2 u_{x_{1}}+3 v_{x_{1}}\right) p_{5}+ \\
& +\left(-u_{x_{3}}+3 w_{x_{1}}+2 v u_{x_{1}}-3 v v_{x_{1}}-v_{x_{3}}+u v_{x_{1}}\right) p_{3}+ \\
& +\left(u u_{x_{3}}-2 u w_{x_{1}}+2 w u_{x_{1}}-3 w v_{x_{1}}-w u_{x_{1}}-u_{x_{2}} v+u_{x_{5}}-w_{x_{3}}\right) p_{1} .
\end{aligned}
$$

Правая часть равенства (4.7) обращается на Г в нуль, если

$$
\begin{aligned}
2 u_{x_{1}}-3 v_{x_{1}} & =0, \\
u_{x_{3}}-3 w_{x_{1}}+v_{x_{3}}-u v_{x_{1}} & =0, \\
u_{x_{5}}+u u_{x_{3}}-2 u w_{x_{1}}-u_{x_{1}} w-v u_{x_{3}}-w_{x_{3}} & =0 .
\end{aligned}
$$

Из первого уравнения, как обычно, имеем $v=2 u / 3$. Таким образом, мы получаем систему

$$
\begin{array}{r}
w_{x_{1}}+\frac{1}{9}(u)_{x_{1}}^{2}-\frac{5}{9} u_{x_{3}}=0, \\
u_{x_{5}}-\frac{7}{9} u u_{x_{3}}+\frac{4}{9} u^{2} u_{x_{1}}-u_{x_{1}} w-w_{x_{3}}=0
\end{array}
$$


или, что эквивалентно, уравнение

$$
\frac{9}{5} u_{x_{5}}-u u_{x_{3}}+u^{2} u_{x_{1}}-u_{x_{1}} \partial_{x_{1}}^{-1} u_{x_{3}}-\partial_{x_{1}}^{-1} u_{x_{3} x_{3}}=0,
$$

которое есть просто уравнение бКП типа Б (бКПБ) [9], [18]. Аналогичным образом можно получить всю иерархию бКПБ, которая представляет собой коизотропные деформации тройных систем Йордана (4.3).

Структурные константы имеют следующий, аналогичный случаю бКП (2.1), вид:

$$
\begin{aligned}
C_{i k l}^{m}=\delta_{i+k+l+1}^{m}+H_{2(k+l-m)+1}^{2 i+1}+H_{2(i+l-m)+1}^{2 k+1}+H_{2(i+k-m)+1}^{2 l+1}+ \\
+\sum_{\substack{p, t=0 \\
p+t=l-1-m ; m \geqslant 0}}^{\infty} H_{2 p+1}^{2 i+1} H_{2 t+1}^{2 k+1}+\sum_{\substack{p, s=0 \\
p+s=k-1-m ; m \geqslant 0}}^{\infty} H_{2 p+1}^{2 i+1} H_{2 s+1}^{2 l+1}+ \\
+\sum_{\substack{t, s=0 \\
t+s=i-1-m ; m \geqslant 0}}^{\infty} H_{2 t+1}^{2 k+1} H_{2 s+1}^{2 l+1},
\end{aligned}
$$

где $H_{2 p+1}^{2 i+1}=0$ при $p \leqslant 1$. Условия ассоциативности (4.5) эквивалентны бесконечному множеству кубических уравнений для коэффициентов $H_{2 p+1}^{2 i+1}$, а условия коизотропности эквивалентны этим алгебраическим условиям ассоциативности и уравнениям точности, аналогичным тем, которые мы имели в случае бКП.

Двухточечная иерархия бКП с инволюцией $p_{i} \rightarrow-q_{i}, q_{i} \rightarrow-p_{i}$ дает нам еще один интересный пример. В этом случае мы имеем два семейства соотношений (4.1), а именно

$$
\begin{aligned}
p_{2 i+1} & =\sum_{k=0}^{i} v_{i, k}(x, y) p_{1}^{2 k+1}, \\
q_{2 i+1} & =\sum_{k=0}^{i} w_{i, k}(x, y) q_{1}^{2 k+1}
\end{aligned}
$$

со склеивающим соотношением

$$
p_{1} q_{1}=u(x, y)
$$

где $(x, y)=\left(x_{1}, x_{3}, x_{5}, \ldots ; y_{1}, y_{3}, y_{5}, \ldots\right)$.

Соответствующая замкнутая "алгебра" определяется следующей таблицей умножения:

$$
\begin{aligned}
p_{i} p_{k} p_{l} & =\sum_{m=1} A_{i k l}^{m}(x, y) p_{m}, \\
q_{i} q_{k} q_{l} & =\sum_{m=1} B_{i k l}^{m}(x, y) q_{m}, \\
p_{i} p_{k} q_{l} & =\sum_{m=1} C_{i k l}^{m}(x, y) p_{m}+\sum_{m=1} D_{i k l}^{m}(x, y) q_{m}, \\
p_{i} q_{k} q_{l} & =\sum_{m=1} E_{i k l}^{m}(x, y) p_{m}+\sum_{m=1} F_{i k l}^{m}(x, y) q_{m} .
\end{aligned}
$$


Формулы (4.12) определяют двойную тройную систему Йордана. В параметризации (4.10), (4.11) структурные константы $A$ и $B$ определяются соотношениями типа (4.2), в то время как

$$
\begin{array}{llll}
C_{111}^{1}=u, & D_{111}^{m}=0, & E_{111}^{m}=0, & F_{111}^{1}=u, \\
C_{113}^{1}=w_{1,1} u, & D_{113}^{1}=u^{2}, & E_{311}^{1}=u^{2}, & F_{311}^{1}=u v_{1,1}
\end{array}
$$

и т.Д.

Вообще "структурные константы” $A, B, C, D, E, F$ удовлетворяют набору кубических условий "ассоциативности". Условие коизотропности для гамильтонианов, определяемых формулами (4.12), снова порождает интегрируемые деформации тройной системы (4.12). Они могут быть названы двухточечной иерархией бКПБ.

Рассмотрим совокупность трех низших гамильтонианов из формул (4.10), (4.11), т.e.

$$
\begin{gathered}
f=p_{3}-p_{1}^{3}+v p, \\
\tilde{f}=q_{3}-q_{1}^{3}+w q_{1}, \\
H=p_{1} q_{1}-u(x, y) .
\end{gathered}
$$

Условия коизотропности для нулевого множества $\Gamma: f=g=H=0$ принимают вид

$$
\begin{aligned}
\{f, \tilde{f}\} & =3\left(w_{x_{1}} p_{1}-v_{y_{1}} q_{1}\right) H, \\
\{f, H\} & =v_{x_{1}} H, \\
\{\tilde{f}, H\} & =w_{y_{1}} H
\end{aligned}
$$

вместе с уравнениями

$$
\begin{aligned}
u_{x_{3}}+(u v)_{x_{1}} & =0, \\
v_{y_{1}}-3 u_{x_{1}} & =0, \\
u_{y_{3}}+(u w)_{y_{1}} & =0, \\
w_{x_{1}}-3 u_{y_{1}} & =0 .
\end{aligned}
$$

Эта система представляет собой бездисперсионный предел уравнения НижникаВеселова-Новикова (уравнение бНВН) [10] (для решений вида $u=u\left(x_{1}, y_{1}, x_{3}+y_{3}\right)$, если точнее). Все семейство коизотропных деформаций для алгебры (4.12) дается иерархией бНВН.

Основные системы для рассмотренных выше алгебраических структур, существование тау-функций и соответствующих бездисперсионных уравнений Хироты будут рассмотрены в последующих работах.

\section{5. СТАЦИОНАРНЫЕ РЕДУКЦИИ БЕЗДИСПЕРСИОННЫХ ИЕРАРХИЙ И ДИНАМИЧЕСКИЕ СИСТЕМЫ, ИНТЕГРИРУЕМЫЕ НА ФИКСИРОВАННОМ УРОВНЕ ЭНЕРГИИ}

Интегрируемые иерархии и в целом конструкция, рассмотренная в настоящей работе, допускают редукции, для которых некоторые переменные $x_{i}$ являются циклическими. Например, в случае иерархии бКП цикличность переменной $x_{2}$ сводит ее 
к бездисперсионной иерархии Кортевега-де Фриза. При такой редукции $\left\{p_{i}, f\right\}=0$ для любой функции $f$ и, следовательно, $p_{i}=$ const. Таким образом, число канонических переменных эффективно уменьшается вдвое, и число гамильтонианов становится равным половине от полного числа переменных.

Проанализируем такую ситуацию более внимательно. Начнем с уравнения бКП и предположим, что переменная $x_{3}$ является циклической, следовательно, $p_{3}=$ const. При такой стационарной редукции уравнение бКП принимает вид

$$
\frac{\partial^{2} u}{\partial x_{2}^{2}}+\frac{\partial^{2} u^{2}}{\partial x_{1}^{2}}=0
$$

и мы имеем два гамильтониана (1.10):

$$
\begin{aligned}
H & :=h_{2}=-p_{2}+p_{1}^{2}+u\left(x_{1}, x_{2}\right), \\
H_{1} & :=h_{3}=p_{1}^{3}+\frac{3}{2} u p_{1}+v+\text { const }
\end{aligned}
$$

где $\partial v / \partial x_{1}=(4 / 3) \partial u / \partial x_{2}$; они находятся в инволюции: $\left\{H, H_{1}\right\}=0$.

Динамическая система, соответствующая гамильтониану $H$, содержит, в частности, уравнения

$$
\begin{aligned}
& \frac{\partial x_{1}}{\partial t}=2 \frac{\partial H}{\partial p_{1}}=2 p_{1} \\
& \frac{\partial p_{1}}{\partial t}=-\frac{\partial H}{\partial x_{1}}=-\frac{\partial u}{\partial x_{1}}
\end{aligned}
$$

и, следовательно,

$$
\frac{\partial^{2} x_{1}}{\partial t^{2}}=-2 \frac{\partial u}{\partial x_{1}}
$$

Функция $H_{1}$ является для динамической системы (5.3) интегралом движения, кубическим по импульсу. Это наблюдение воспроизводит результат работы [19], утверждающий, что динамическая система $(5.3),(5.4)$ с потенциалом $u\left(x_{1}, x_{2}\right)$, удовлетворяющим уравнению (5.1), имеет дополнительный интеграл движения, кубический по импульсу $p_{1}$.

В случае цикличности по переменным $x_{n}(n \geqslant 4)$ мы имеем гамильтониан $H_{n}=$ $p_{1}^{n}+\cdots$, находящийся в инволюции с гамильтонианом $H$, который является интегралом движения для системы (5.3) порядка $n$ [19].

Другой простой пример дает стационарная система бНВН (4.14), (4.15). Чтобы установить связь с обычными двумерными динамическими системами, наложим ограничения $y_{1}=\bar{x}_{1}, q_{1}=\bar{p}_{1}, q_{3}=\bar{p}_{3}, w=\bar{v}$ (черта означает комплексное сопряжение) и предположим, что $u$ зависит только от переменных $x_{1}, \bar{x}_{1}$ и $t=x_{3}+y_{3}$. Переходя к новым переменным $x_{1} \rightarrow z:=x_{1}+i x_{2}, p_{1} \rightarrow p:=p_{1}+i p_{2}$, можно переписать гамильтониан $H$ в виде

$$
H=p_{1}^{2}+p_{2}^{2}+u\left(x_{1}, x_{2}, t\right),
$$


а соответствующее уравнение примет вид

$$
\begin{aligned}
& \frac{\partial u}{\partial t}+\frac{\partial(u v)}{\partial z}+\frac{\partial(u \bar{v})}{\partial \bar{z}}=0 \\
& \frac{\partial v}{\partial \bar{z}}=3 \frac{\partial u}{\partial z}
\end{aligned}
$$

Это есть бездисперсионное уравнение Веселова-Новикова (уравнение бВН) [2], [10]. Вместо двух гамильтонианов $f$ и $\tilde{f}(4.14)$ мы имеем для уравнения (5.6) их сумму

$$
\widetilde{H}=f+\tilde{f}=p_{3}+\bar{p}_{3}-p^{3}+v p-\bar{p}^{3}+\bar{v} \bar{p}
$$

И

$$
\{H, \widetilde{H}\}=\left(v_{z}+\bar{v}_{\bar{z}}\right) H .
$$

Формулы (5.7), (5.8) означают, что для любого решения уравнения бВН (5.6) динамическая система с гамильтонианом $H(5.5)$ обладает на нулевом уровне энергии $E=0$ дополнительным интегралом движения $\widetilde{H}(5.7)$. В частности, в стационарном случае $\partial u / \partial t=0$ сумма $p_{3}+\bar{p}_{3}$ постоянна и, следовательно,

$$
\widetilde{H}=-p^{3}+v p-\bar{p}^{3}+\bar{v} \bar{p}+\text { const. }
$$

Таким образом, в этом случае мы имеем динамическую систему с двумя степенями свободы, которая при нулевом уровне энергии $E=0$ имеет дополнительный кубический интеграл движения (5.9), если потенциал $u$ является решением стационарного уравнения бНВ

$$
\frac{\partial(u v)}{\partial z}+\frac{\partial(u \bar{v})}{\partial \bar{z}}=0, \quad \frac{\partial v}{\partial \bar{z}}=3 \frac{\partial u}{\partial z} .
$$

Такие интегралы движения обычно называются условными, или конфигурационными (см., например, монографии [20]). Связь между существованием условных кубических интегралов (5.9) и уравнением (5.10) была установлена в рамках другого подхода в работе [21] (формула (7.5.12)). Аналогично высшие условные интегралы нечетного порядка для гамильтоновой системы (5.5), найденные в работе [21], являются гамильтонианами $H_{n}$, связанными с высшими стационарными уравнениями бВН.

Таким образом, стационарные иерархии бКП, бВН и другие стационарные бездисперсионные интегрируемые иерархии предоставляют нам динамические системы, вполне интегрируемые по Лиувиллю на фиксированном уровне энергии.

Полные бездисперсионные иерархии в нашей схеме характеризуются существованием такого числа независимых гамильтонианов, которое равно половине размерности симплектического пространства минус один. Они представляют собой иерархии, наиболее близкие к вполне интегрируемым по Лиувиллю иерархиям, и их можно назвать почти полностью интегрируемыми по Лиувиллю.

Благодарности. Авторы выражают благодарность А. Моро за помощь в подготовке статьи. Работа была частично поддержана грантами COFIN 2004 "Sintesi" и COFIN 2004 "Nonlinear Waves and Integrable systems". 


\section{Список литературы}

[1] В.Е. Захаров, Функи. анализ и его прилож., 14:2 (1980), 15-24; Y. Kodama, Phys. Lett. A, 129 (1988), 223-226; Б. А. Дубровин, С. П. Новиков, УМН, 44:6 (1989), 29-98; B. A. Kuperschmidt, J. Phys. A, 23 (1990), 871-886; R. Carroll, Y. Kodama, J. Phys. A, 28 (1995), 6373-6387; J. Gibbons, S. P. Tsarev, Phys. Lett. A, 211 (1996), 19-24; P. B. Wiegmann, A. Zabrodin, Commun. Math. Phys., 213 (2000), 523-538.

[2] И. М. Кричевер, Функи. анализ и его прилож., 22:3 (1988), 37-52.

[3] Y. Kodama, Phys. Lett. A, 147 (1990), 477-482.

[4] K. Takasaki, T. Takebe, Internat. J. Modern Phys. A, 7 (1992), 889-922.

[5] K. Takasaki, T. Takebe, Rev. Math. Phys., 7 (1995), 743-808.

[6] I. M. Krichever, Commun. Pure Appl. Math., 47 (1994), 437-475.

[7] V.E. Zakharov, "Dispersionless limit of integrable systems in $2+1$ dimensions", Singular Limits of Dispersive Waves, Proc. NATO Adv. Res. Workshop (Lyon, France, 1991), NATO Adv. Sci. Inst. Ser. B, Phys., 320, eds. N. M. Ercolani et al., Plenum, New York, 1994, 165174 .

[8] A. Boyarsky, A. Marshakov, O. Ruchayskiy, P. B. Wiegmann, A. Zabrodin, Phys. Lett., 515 (2001), 483-492.

[9] B. G. Konopelchenko, L. Martinez Alonso, J. Math. Phys., 43 (2003), 3807-3823.

[10] B. G. Konopelchenko, L. Martínez Alonso, Stud. Appl. Math., 109 (2002), 313-336.

[11] B. G. Konopelchenko, F. Magri, Coisotropic deformations of associative algebras and dispersionless integrable hierarchies, nlin.SI/0606069.

[12] A. Weinstein, J. Math. Soc. Japan, 40 (1988), 705-727; R. Berndt, An Introduction to Symplectic Geometry, Grad. Stud. Math., 26, AMS, Providence, RI, 2001.

[13] G. Falqui, F. Magri, M. Pedroni, Commun. Math. Phys., 197 (1998), 303-324.

[14] E. Witten, Nuclear Phys. B, 340 (1990), 281-332; R. Dijkgraaf, H. Verlinde, E. Verlinde, Nuclear Phys. B, 352 (1991), 59-86.

[15] L. C. Li, Commun. Math. Phys., 203 (1999), 573-592.

[16] M. Błaszak, Phys. Lett. A, 297 (2002), 191-195.

[17] N. Jacobson, Structure and Representation of Jordan Algebras, Colloq. Publ., 39, AMS, Providence, RI, 1968; E. Neher, Jordan Triple Systems by the Grid Approach, Lect. Notes Math., 1280, Springer, Berlin, 1987.

[18] K. Takasaki, Lett. Math. Phys., 28 (1993), 177-185.

[19] В. В. Козлов, Матем. заметки, 45:4 (1989), 46-52.

[20] G. D. Birkhoff, Dynamical Systems, AMS, New York, 1927; E. T. Whittaker, A Treatise on the Analytical Dynamics of Particles and Rigid Bodies with an Introduction to the Problem of Three Bodies, Cambridge Univ. Press., Cambridge, 1937.

[21] J. Hietarinta, Phys. Rep., 147 (1987), 87-154. 